\title{
A delayed damage model for the prediction of dynamic fracture experiments
}

\author{
Application on metallic structures
}

\author{
Arnaud Suffis* — Alain Combescure** _ Pierre Chevrier *** \\ * Laboratoire d'Etude de Dynamique Commissariat a l'Energie Atomique - Saclay \\ F-91191 Gif-Sur-Yvette cedex \\ ** LaMCoS INSA-Lyon UMR CNRS 5259 \\ 18-20 Allée des sciences F-69621 Villeurbanne \\ *** Laboratoire de Physique et de Mecanique des Materiaux \\ Universite de Metz \\ Ile de Saulcy, F-57045 Metz cedex 01 \\ alain.combescure@insa-lyon.fr
}

\begin{abstract}
A delayed damage model was recently introduced to avoid artificial localization and mesh dependence in a softening material during a finite element analysis. This model is also interesting for transient applications because it requires only local information to predict damage and plastic strain rates. The physical idea behind this model is that the void growth rate cannot be infinite and hence the damage rate must be bounded. This paper shows that such a model does not require artificial numerical parameters and can be identified using classical spall fracture experiments. It was applied successfully to experiments performed on two aluminum alloys and one titanium alloy. The identification of the delayed damage parameters is presented. The model is applied to a simple numerical experiment which shows clearly that it avoids artificial numerical localization.

RÉSUMÉ. Un modèle de taux de croissance limité de l'endommagement est proposé pour éviter la localisation artificielle et la dépendance au maillage lors de la simulation numérique de la rupture de matériaux adoucissants par éléments finis. Ce type de modèle est spécialement intéressant pour les analyses dynamiques car il ne nécessite pas d'informations non locales pour éviter la localisation. Le modèle repose sur une idée physique très simple: l'endommagement ne peut croître à une vitesse infinie. Le modèle est identifié sur deux alliages d'aluminiums et un titane. Une expérimentation numérique de rupture dynamique sous choc montre que ce type de modèle évite la localisation numérique.

KEYWORDS: identification, delayed damage, plate impact, spalling, finite element, numerical localization.

MOTS-CLÉS: identification, endommagement retardé, impacts plaque plaque, écaillage, éléments finis, localisation numérique.
\end{abstract}

DOI:10.3166/REMN.16.601-625 C Lavoisier, Paris 


\section{Introduction}

One of the main difficulties encountered in the numerical simulation of ductile failure is that because of the softening behavior of the material the calculated solution is mesh-dependent. Thus, numerical predictions do not converge when the mesh size tends to zero.

Simulations lead to artificial localization of the deformations, which are concentrated in a single element (usually the smallest one). In statics, this problem is solved by introducing the physical concept of characteristic length. This length enables one to take stresses or damage in this characteristic zone into account in an average sense in order to predict behavior: these are non-local models.

Second-gradient theories, which introduce strain gradients into the constitutive relation, also enable one to suppress mesh dependence. These theories remove this dependence at the cost of auxiliary calculations: while non-local models involve only calculations in the vicinity of the elements concerned, second-gradient methods require the resolution of a second linear system whose size is equivalent to that of the standard structural analysis.

Of course, when attempting to predict fracture in dynamics using numerical simulation, one can still use such non-local models, but this requires significant modifications in standard software and makes an already computer-intensive calculation even more time consuming. The approach developed in this paper consists in starting from the models which control the damage rate. The physical concept is extremely easy to understand: cavities simply cannot expand at an infinite rate.

These models also enable one to perform some sort of average over the time variable instead of the space variable. This idea was already introduced in Seaman's models (Seaman et al., 1987) for the DEFRACT program. However, one drawback of such models is their purely dynamic formulation which requires a complete dynamic identification through dynamic tests: results from static characterization can no longer be used. The idea behind the model presented here is simply to complement a traditional damage model with an equation controlling the damage rate (Ladevèze, 1992; Deu et al., 1997; Allix et al., 1999; Ladevèze et al., 2000; Suffis et al., 2002; 2003; Suffis, 2004): this approach enables one to identify the constitutive laws in statics, then to add an identification step for the dynamic part of the model. The parameters controlling the damage rate are identified using plateplate impact tests. This paper shows how the method was developed for three materials and presents the results of the identification for two aluminum alloys: 7020-T6 and AU4G1-T4. The static rupture and plate-plate impact tests can be found in References (Chevrier, 1998; Chevrier et al., 1999). An interesting reference (Ikkurthi et al., 2004) compares several damage models in dynamics, including the DFRACT model, and points out the good quality of the predictions obtained from models with controlled growth. 
In this paper, we chose to use the Chaboche Lemaitre model (Chaboche et al., 1996; Lemaitre, 1996).

First, the paper describes the equations of the model and reviews the main theoretical results already available. Then, it presents the principle of the plate-plate tests. The identification is developed based on the results of these tests. Finally, the model with controlled damage rate is compared with the model without control in the case of the simulation of a shear fracture test: this example demonstrates the advantage of our model in the controlled prediction of the fracture mode.

\section{The delayed damage model}

The damage models commonly used in numerical analysis suffer from mesh dependence and usually lead to artificial strain localization within a single element. The delayed damage model introduced by (Ladevèze, 1992; Deu et al., 1997; Ladevèze et al., 2000) for composite laminates and later extended by (Suffis et al., 2002; Suffis et al., 2003) to metals enables one to avoid this mesh dependence.

\subsection{Mesh independence}

It is now well-established that the numerical prediction of the failure of a softening material is mesh-dependent (Suffis et al., 2003; Suffis, 2004). For transient problems, a simple solution consists in introducing a "delay effect" into the damage evolution law. The damage rate is then determined through the fundamental equation of the delayed damage model, which is:

$$
\dot{D}=\frac{1}{\tau_{c}}\left(1-e^{-a\left(D_{N C}-D\right)}\right)
$$

The coefficient a and the characteristic time $\tau_{c}$ are the two parameters of the model. $D_{N C}$ is the original unlimited damage time function. While the standard damage rate tends to infinity as the mesh size tends to zero, the delayed damage model introduces an upper bound on the damage rate (the limit is $\frac{1}{\tau_{c}}$ ). 
Equation [1] has an analytical solution within time step $\Delta \mathrm{t}$ which is:

$$
\Delta D(t)=-\frac{1}{a} \log \left[\frac{e^{-\frac{\Delta D_{N C}}{\Delta t} t}-\frac{\Delta D_{N C}}{\Delta t} \tau_{c} e^{-\frac{a}{\tau_{c}} t}}{1-\frac{\Delta D_{N C}}{\Delta t} \tau_{c}}\right]
$$

Previous work done on this model (Suffis et al., 2003) showed mathematically and numerically that it leads to a mesh-independent solution. In the one-dimensional case of a bar in tension, the size of the fully-damaged zone $L_{c a r}$ is known analytically and given by Equation [3]:

$$
L_{c a r}=c \tau_{c} \ln \left(\frac{\Delta \sigma}{\sigma_{c r i t}}\right)
$$

where $c$ is the velocity of sound $\left(c=\sqrt{\frac{E}{\rho}}\right)$ and $\Delta \sigma$ the elastic stress change corresponding to the prescribed strain rate.

\subsection{Identification of a and $\tau_{c}$}

Having chosen a model which avoids mesh dependence, we must still identify the delayed damage parameters. As outlined in the introduction, the main purpose of the delayed damage model is to limit the damage rate. This limitation is particularly crucial for very severe loads and short loading times, in which case damage evolves very rapidly. According to D.R. Curran, L. Seaman and D.A. Shockey (Curran et al., 1987), the plate impact experiment has these characteristics. Since the loading time is very short (a few microseconds), this type of impact should constitute a good test in order to evaluate the influence of the delayed damage parameters. The plate impact experiment is described in the next section and the results on two aluminum alloys along with the identification of the parameters are given in Section 5.

\section{Description of the plate impact experiment}

\subsection{Main characteristics}

The plate impact experiment consists in launching a thin plate of thickness $l_{i}$ and diameter $\mathrm{d}$, called the impactor, towards a flat stationary target plate with the same 
diameter and thickness $l_{c}$ at a prescribed velocity $\mathrm{V}_{0}$. This test is usually performed with an impactor and a target of the same material and such that:

$$
l_{i}=\frac{l_{c}}{2}
$$

If the velocity is small enough that stresses do not exceed Hugoniot's elastic limit, the incident wave is purely elastic. If the velocity is higher, an elastic precursor followed by slower plastic waves propagate within both the impactor and the target. The waves, which are initially compressive waves, reflect on the free surface of the plates and become tensile waves. If the plates are chosen as specified above, the two waves meet in the center of the target and lead to a tensile stress state. If the amplitude and duration of the stress in the center of the target are sufficient, the tension state leads to spalling (i.e. a new surface) at that location. These successive steps are described by the phase diagram in Figure 1.

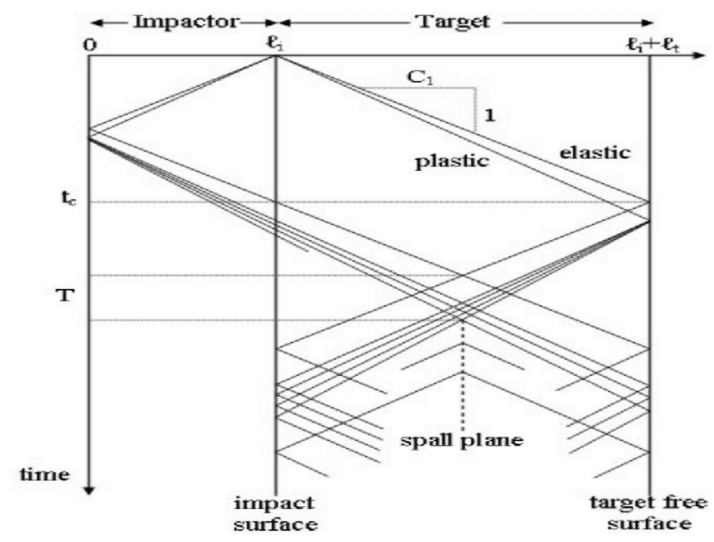

Figure 1. Schematic space time diagram: the waves crossing in the middle of the target create a tensile stress state

Such an experiment requires sophisticated equipment (launcher, sensors) and enables one to measure several quantities. This is the topic of the following section.

\subsection{The experiment}

The experiment described here was performed at the LPMM (Chevrier et al., 1994; Chevrier, 1998). This laboratory is equipped with a $57 \mathrm{~mm}$ diameter gas launcher for plate impact capable of sending the projectile towards the target at a maximum velocity of $600 \mathrm{~m} . \mathrm{s}^{-1}$. 
An optical system consisting of three laser beams is used to measure the impact velocity of the projectile. A laser interferometer monitors the evolution of the velocity of the rear free surface of the target, called the pull-back velocity. A specially designed catcher carefully recovers the two plates following the impact to prevent any secondary damage.

The critical time $t^{c}$ and the applied stress $\sigma^{S}$ are given by the following formulae:

$$
\begin{aligned}
& t^{c}=\frac{2 l_{i}}{C_{1}} \\
& \sigma^{S}=\rho C_{1} \frac{V_{0}}{2}
\end{aligned}
$$

These quantities depend only on the thickness $l_{i}$ and the velocity $\mathrm{V}_{0}$. In these equations, $\rho$ is the density of the material being considered and $\mathrm{C}_{1}$ is the longitudinal elastic wave velocity, which is given by:

$$
C_{1}=\sqrt{\frac{E(1-v)}{\rho(1+v)(1-2 v)}}
$$

where E is the Young's modulus, $v$ the Poisson's ratio and $\rho$ the density.

The history of the velocity of the rear impact face is used to determine the degradation of the target. In order to check the degradation state of the specimens, the impacted target is cut along a plane perpendicular to the impact direction after each test. The surfaces are polished, etched with marble and nital solutions and examined under an optical microscope in order to determine the spall level (Chevrier 1998). This level ranges from "no spall" (for a material with no microcavity or microcrack visible up to a magnification factor of 1000) to "complete spall" for a specimen with a new free surface. A spalled specimen is shown in Figure 2. For a given thickness $l_{i}$, it is possible to determine the spall stress (i.e. the applied stress at which spalling occurs) by conducting successive experiments with increasing velocities. Finally, the curve giving $\sigma^{S}$ as a function of $t^{c}$ can be obtained by using a series of specimens with different thicknesses: the resulting curve for aluminum alloy 7020-T6 is shown in Figure 6 

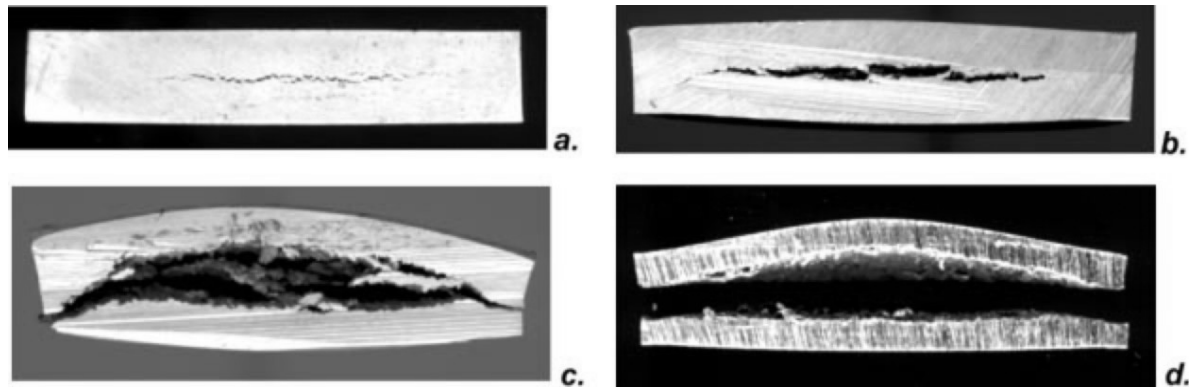
a) $150 \mathrm{~m} / \mathrm{s} t^{c}=1.7 \mu \mathrm{s}$
b) $256 \mathrm{~m} / \mathrm{s} t^{c}=1.7 \mu \mathrm{s}$
c) $270 \mathrm{~m} / \mathrm{s} t^{c}=1.7 \mu \mathrm{s}$
d) $\left.294 \mathrm{~m} / \mathrm{s} t^{c}=1.0 \mu \mathrm{s}\right]$

Figure 2. Aluminum alloy targets as recovered after impact tests at four different velocities

Experimental results of this type show that the shorter the critical time, the higher the spall stress. (Chevrier, 1998; Chevrier et al., 1999) proposed an interpretation of this behavior using a model based on a cumulative fracture criterion. An alternative interpretation is based on the combination of a viscoplastic damage model (described in Section 4) followed by a delayed damage model (see Section 5).

\section{The materials studied}

Three materials are considered in this paper: aluminum alloy 7020 (Mn $0.05 \%$, Mg $1 \%$, Cr $0.1 \%$, Zn $4 \%$ ) with T6 treatment, AU4G1-T4 aluminum, and a special TA6V titanium alloy. Several simple tension and compression tests were performed to determine the mechanical characteristics of these materials (Chevrier, 1998; Chevrier et al., 1999; Hanim et al., 1999), which are summarized in Table 1:

Table 1. Basic material properties of the two aluminum materials

\begin{tabular}{|l|c|c|c|c|}
\hline material & $\boldsymbol{\rho}$ & $\sigma^{y}$ & $\mathbf{E}$ & $\mathbf{C}_{1}$ \\
\hline & $\mathrm{kg} \cdot \mathrm{m}^{-3}$ & $\mathrm{MPa}$ & $\mathrm{MPa}$ & $\mathrm{m}^{-\mathrm{s}^{-1}}$ \\
\hline AL 7020 T6 & 2,780 & 320 & 71,500 & 5,883 \\
\hline AU4G1 T4 & 2,820 & 314 & 74,000 & 5,942 \\
\hline
\end{tabular}


In Table $1, \rho, \sigma^{y}, \mathrm{E}$ and $\mathrm{C}_{1}$ are respectively the density, the yield stress, the Young's modulus and the longitudinal elastic wave velocity. A moderate strain rate dependence was found with relatively little hardening. Damage and rupture were modeled by introducing an isotropic damage variable $D$. When a material is subjected to huge strain rates, its Young's modulus often depends on the strain rate, but this effect was not investigated.

\subsection{General equations}

The three materials were assumed to follow the rate-dependent J2 (Huber-Mises) flow theory of plasticity with isotropic hardening coupled with damage.

The total strain tensor $\underset{\underline{\varepsilon}}{\underline{E}}$ can be decomposed into an elastic part and a plastic part.

$$
\underline{\underline{\varepsilon}}=\underline{\underline{\varepsilon^{e}}}+\underline{\underline{\varepsilon^{p}}}
$$

The stress tensor $\underline{\underline{\sigma}}$ (which corresponds to the quantity measured macroscopically) is related to the effective stress tensor $\underline{\underline{\sigma^{e f f}}}$ (which corresponds to a microscopic quantity) through the damage variable D.

$$
\underline{\underline{\sigma^{e f f}}}=\frac{\underline{\underline{\sigma}}}{1-D}
$$

Damage goes from 0 to a critical value $D^{c}$, at which rupture occurs. In the calculation, when this critical value is attained within an element, the Young's modulus of this element is drastically decreased.

The Hooke's law is:

$$
\underline{\underline{\sigma}}=(1-D) \underline{\underline{H}} \underline{\underline{\varepsilon^{e}}}
$$

where $\underline{\underline{H}}$ is the Hooke's matrix. The rates of the different mechanical quantities are given by Equations [11] to [15].

$$
\begin{aligned}
& \underline{\dot{\varepsilon}}=\underline{\underline{\dot{\varepsilon}^{e}}}+\underline{\underline{\dot{\varepsilon}^{p}}} \\
& \underline{\underline{\sigma}}=(1-D) \underline{\underline{H}} \underline{\underline{\dot{\varepsilon}^{e}}-\frac{\dot{D}}{1-D}} \underline{\underline{\sigma}}
\end{aligned}
$$




$$
\begin{aligned}
& \underline{\underline{\dot{\varepsilon}^{p}}}=\frac{3}{2} \frac{\lambda}{1-D} \underline{\underline{\underline{\sigma^{*}}}} \\
& \dot{p}(1-D)=\lambda \\
& \dot{D}=h\left(\frac{\sigma^{H}}{\sigma^{*}}, D\right) \dot{p}
\end{aligned}
$$

In these equations, $\lambda$ represents the plastic multiplier, $\underline{\underline{\sigma^{d}}}$ the deviatoric part of the stress tensor, $\sigma^{*}$ the Von Mises' stress, $\mathrm{p}$ the equivalent plastic strain and $\sigma^{H}$ the hydrostatic stress. The damage rate (Equation [15]) depends on the triaxiality $\left(\frac{\sigma^{H}}{\sigma^{*}}\right)$ and the damage rate $\dot{D}$ and is proportional to the equivalent plastic strain rate $\dot{p}$. This model is simply the ductile plastic model developed in (Chaboche 1996).

Finally, the yield function is defined by Equation [16]:

$$
\begin{aligned}
& f(\underline{\underline{\sigma}, D}, p, \dot{p}, T)=\frac{\sigma^{*}}{1-D}-\sigma^{y}(p, \dot{p}, T) \\
& p=\int_{0}^{t} \dot{p} d t, \dot{p}=\sqrt{\frac{2}{3} \underline{\underline{\dot{\varepsilon}^{p}} \otimes{\dot{\dot{\varepsilon}^{p}}}^{\underline{\underline{y}}}}}
\end{aligned}
$$

where $\sigma^{y}$ is the yield stress, which depends on the equivalent plastic strain $\mathrm{p}$, the equivalent plastic strain rate $\dot{p}$ and the temperature T. This is explained in the next section.

\subsection{Strain rate dependence}

Aluminum AU4G1-T4 is not strain-rate-dependent. The stress-strain law is described in Figure 3. 
610 Revue européenne de mécanique numérique. Volume 16 - n 5/2007

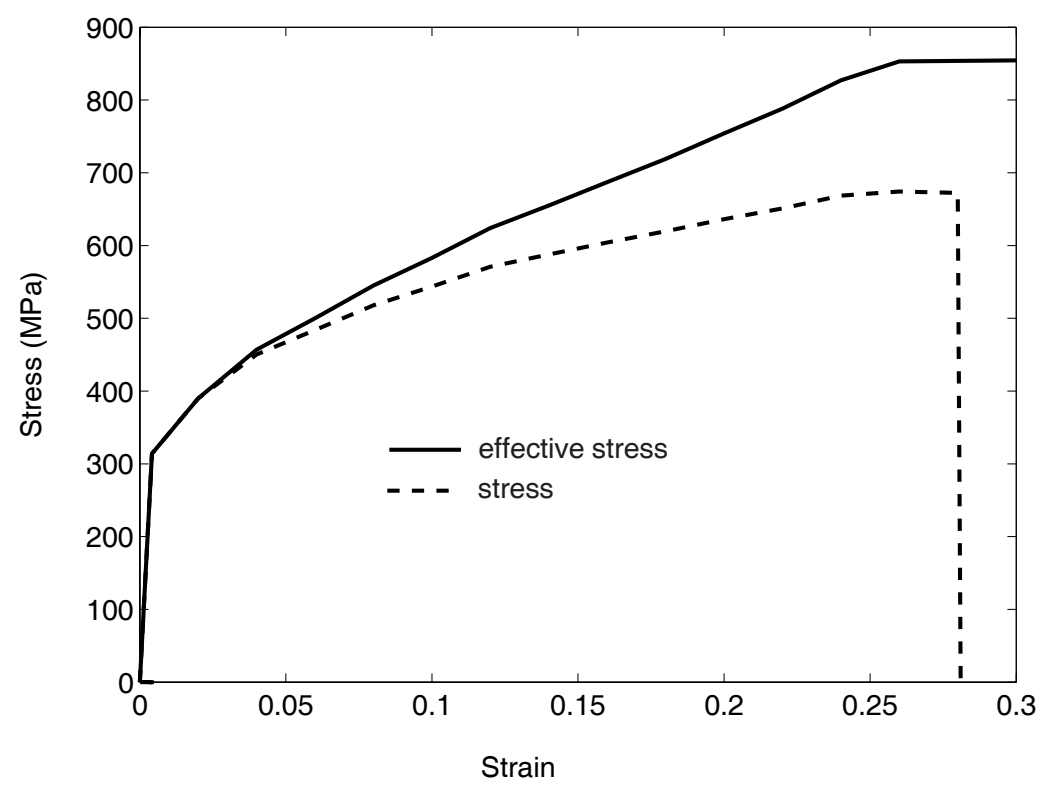

Figure 3. Stress-strain curve for Au4G T4

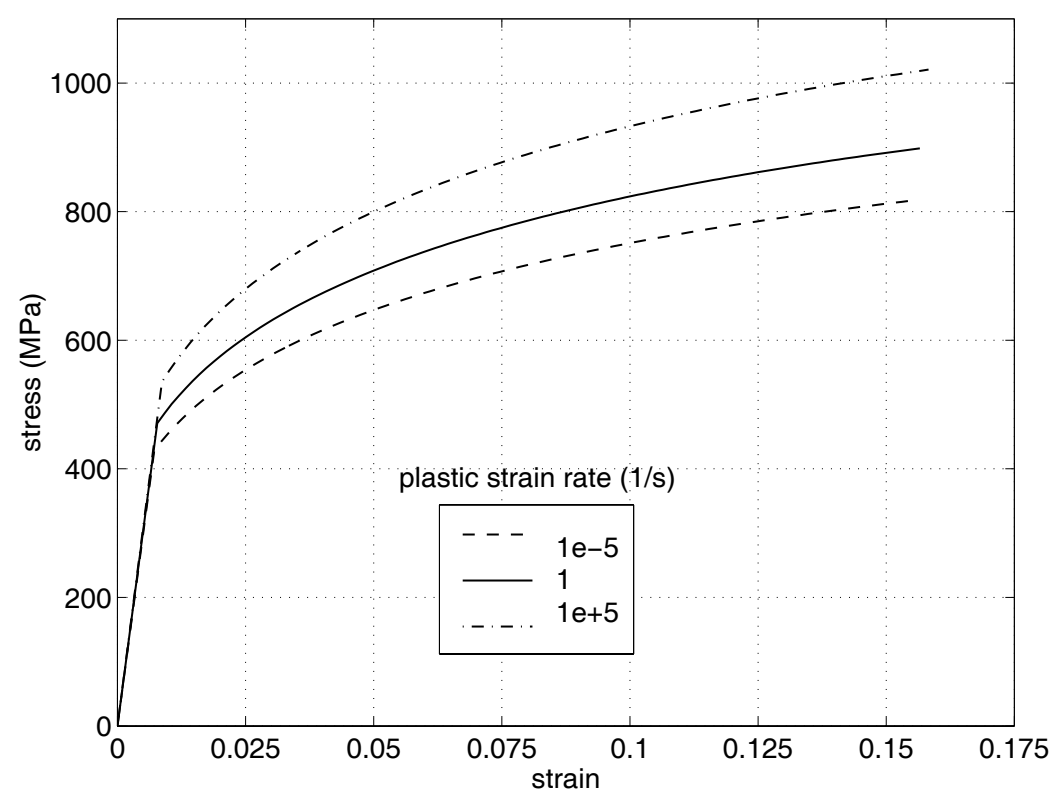

Figure 4. Stress-strain curves for Al 7020 T6 at different strain rates 
The stress-strain curve of aluminum alloy 7020-T6 is strain-rate-dependent and was identified using tensile and compressive tests at different strain rates: the corresponding curves are shown in Figure 4. The flow stress is assumed to follow a power law of the form given by Equations [18] to [21]:

$$
\sigma^{y}(p, \dot{p}, T)=\sigma_{0}(p, T)\left(1+\left(\frac{\dot{p}}{\dot{p}_{0}}\right)^{m(T)}\right)
$$

with

$$
\sigma_{0}(p, T)=B_{0}\left(1-q \frac{T}{T_{m}}\right)\left(p+p_{0}\right)^{n(T)}
$$

and

$$
\begin{aligned}
& n(T)=n_{0}\left(1-\frac{T}{T_{m}}\right) \\
& m(T)=m_{0} \frac{T}{T_{m}}
\end{aligned}
$$

$\sigma_{0}(p, T)$ represents the flow stress under quasi-static conditions, which is described by a power law of the equivalent plastic strain $\mathrm{p}$ and varies linearly with respect to the absolute temperature T. $B_{0}$ is the plastic modulus, $p_{0}$ the reference plastic strain and $n_{0}$ the strain hardening exponent. Under dynamic conditions, the quasi-static flow stress is multiplied by a power function of the equivalent plastic strain rate $\dot{p} \cdot \dot{p}_{0}$ is the reference equivalent strain rate and $m_{0}$ the strain rate sensitivity. Finally, $\mathrm{q}$ is a coefficient describing the temperature sensitivity and $T_{m}$ a reference temperature. These equations were proposed by (Klepaczko, 1987). The material constants $B_{0}, q, p_{0}$ and $\mathrm{n}$ were determined by (Hanim, 1999) by fitting an experimental true-stress/true-strain curve obtained at a strain rate $\dot{p}$ equal to $11.3 \mathrm{~s}^{-1}$. The other coefficients $m_{0}$ and $p_{0}$ accounting for the rate sensitivity were determined by the same authors using a linear form of Equation [18]:

$$
\log (\dot{p})=\left(\frac{1}{m(T)}\right) \log \left[\frac{\sigma}{\sigma_{0}}-1\right]+\log \left[\dot{p}_{0}\right]
$$


All the constants for Klepaczko's model were identified by (Hanim, 1999) and are summarized in Table 2.

Table 2. Values of the constants of Klepaczko's model

\begin{tabular}{|c|c|c|c|c|c|c|}
\hline$B_{0}$ & $\mathrm{q}$ & $T_{m}$ & $n_{0}$ & $m_{0}$ & $\dot{\varepsilon}_{0}$ & $\varepsilon_{0}$ \\
\hline $\mathrm{MPa}$ & 1 & ${ }^{\circ} \mathrm{K}$ & 1 & 1 & $s^{-1}$ & 1 \\
\hline 1352 & 1.118 & 1877 & 0.289 & 0.2248 & $1.88 \mathrm{e}+12$ & 0.007 \\
\hline
\end{tabular}

\subsection{Damage}

The damage evolution law $h\left(\frac{\sigma^{H}}{\sigma^{*}}, D\right)$ (Equation [15]) belongs to the family of damage coupled with plasticity models described by (Lemaitre, 1996; Chaboche et al., 1996). The damage evolution law and the associated condition are:

$$
\begin{gathered}
\dot{D}=h\left(\frac{\sigma^{H}}{\sigma^{*}}, D\right) \dot{p}, \quad \text { with } h=\frac{D^{c}}{p^{c}-p^{d}} \Omega\left(\frac{\sigma^{H}}{\sigma^{*}}\right) \text { if } p \Omega\left(\frac{\sigma^{H}}{\sigma^{*}}\right) \geq p^{d} \\
\dot{D}=0 \text { otherwise }
\end{gathered}
$$

where $p^{c}$ and $p^{d}$ are the critical equivalent plastic strain and the threshold strain respectively and $\Omega$ is a function of the triaxiality rate $\left(\frac{\sigma^{H}}{\sigma^{*}}\right)$. For the law considered in this paper, if the triaxiality rate is constant during the whole test, damage evolves linearly from 0 up to its critical value $D^{c}$ which corresponds to a fully-damaged state.

Function $\Omega$ is chosen such that it represents the experimental observations. This type of model is rather elementary, but adequate for ductile plastic failure. In the case of fragile failure, such a model is a somewhat crude. The determination of the other parameters $D^{c}, p^{c}$ and $p^{d}$ governing the damage evolution law will be presented next. 


\subsubsection{Damage parameters}

As explained before, when the triaxiality rate is constant, damage evolves linearly from 0 to $D^{c}$. In particular, if $\Omega\left(\frac{\sigma^{H}}{\sigma^{*}}\right)$ is equal to 1 , the damage D increases linearly when the equivalent plastic strain increases from $p^{d}$ to $p^{c}$. This is the case of simple tension tests on cylindrical specimens, in which $\frac{\sigma^{H}}{\sigma^{*}}=\frac{1}{3}$. One can immediately conclude that the critical plastic strain $p^{c}$ corresponds to that found in such a test. The physical mechanisms which govern the evolution of ductile damage are closely connected to the growth and coalescence of microcavities. According to (Chaboche 1996; Lemaitre, 1996), the measurement of the density of such cavities enables one to predict damage initiation (i.e. the threshold of the plastic strain $p^{d}$ ) as well as the value of the final damage $D^{c}$. (Achon, 1994) showed that for aluminum alloys of the 7000 family the cavity growth starts as soon as plasticity occurs. Thus, damage initiates as soon as the equivalent plastic strain becomes positive. Therefore, the threshold plastic strain $p^{d}$ is set to zero. This is not the case for Alloy AU4G1 T4. However, (Achon, 1994] indicated that for this aluminum alloy the void volume fraction leading to fracture occurs for a strain value equal to a few percents. The simple tension tests performed by (Chevrier, 1999) on aluminum alloy 7020-T6 also showed that damage has a limited influence on the material characteristics. Finally, the identified damage model parameters are given in Table 3. One can observe that the critical damage for Al 7020T6 is small. Since its failure strain is relatively small, this material is rather brittle and, therefore, the choice of a ductile damage growth model might not be optimum.

Table 3. Damage parameters

\begin{tabular}{|c|c|c|c|}
\hline Material & $D^{c}$ & $p^{d}$ & $p^{c}$ \\
\hline Al 7020 T6 & 0.05 & 0. & 0.115 \\
\hline AU4G1 T4 & 0.21 & 0.03 & 0.35 \\
\hline
\end{tabular}




\subsubsection{Influence of the triaxiality rate}

The damage rate depends on the triaxiality rate through Function $\Omega$. This function is such that:

$$
\Omega\left(\frac{1}{3}\right)=1
$$

The objective of this section is to choose an appropriate Function $\Omega$. Equation [26] was found to be the most appropriate to fit the experimental results:

$$
\Omega\left(\frac{\sigma^{H}}{\sigma^{*}}\right)=A+B\left(e^{\left(\left\langle\frac{\sigma^{H}}{\sigma^{*}}\right)\right)^{3}}-1\right)
$$

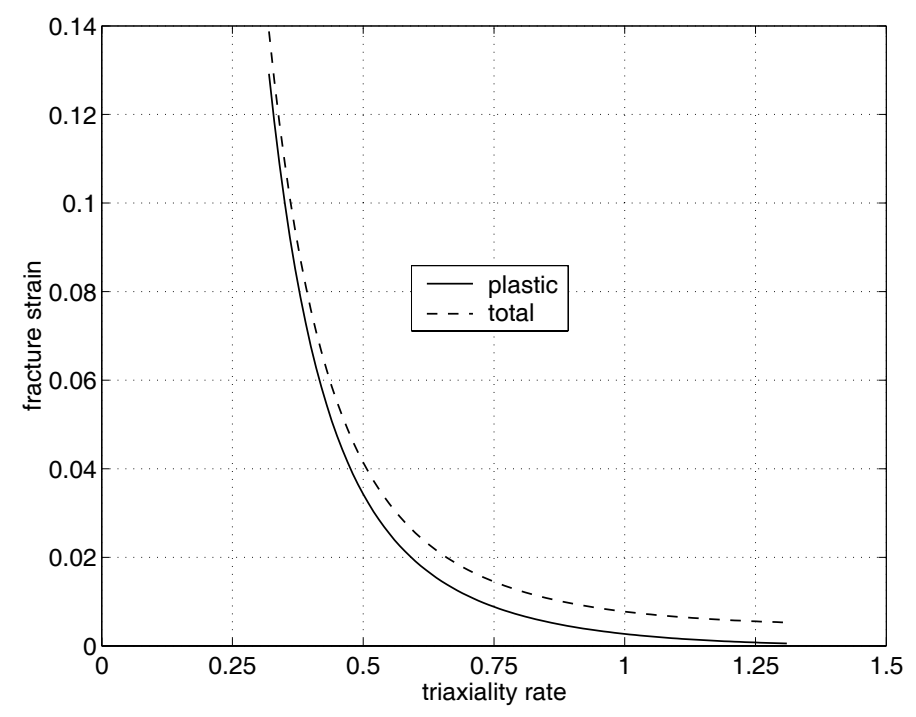

Figure 5. Evolution of the plastic and total fracture strains as functions of the triaxiality ratio

In Equation [26], Coefficients A and B are material constants (whose identified values for aluminum alloy 7020-T6 are 0.0674 and 24.7 respectively) and $\langle\mathrm{x}\rangle$ represents the positive part of $\mathrm{x}$. The proposed function yields $p_{\text {fracture }}=11.5 \%$ and $p_{\text {fracture }}=0.13 \%$ when the triaxiality ratio is equal to $(1 / 3)$ and 1.15 respectively 
(typical values encountered in plate impact results). In addition, one can note that damage evolves very slowly in compression and at small triaxiality rates. It is also possible to deduce the plastic fracture strain associated with a prescribed triaxiality ratio. This evolution is shown in Figure 5. Let us observe that the model presents a triaxiality dependence similar to that of the well-known Gurson's model (Gurson, 1977) for positive triaxialities. For a negative or zero triaxiality ratio, although failure was observed by (Achon 1994) on the aluminum alloy 7000 family, Gurson's model cannot predict rupture. The proposed model is capable of predicting failure also in compression. This aspect is rather important for impact test simulations, in which a significant part of the loading is compressive.

\section{Plate impact simulations}

Let us now focus on the results of the plate impact experiment for this aluminum alloy and on its numerical simulation. First, we will present the results of the simulation of the plate impact experiment using the previous model without delayed damage. Delayed damage was introduced later in order to represent the influence of the loading time on the spall stress properly. At the same time, the experimental results enabled us to identify the parameters of the delayed damage model.

\subsection{Experimental results}

Chevrier 1998; Chevrier et al., 1999) performed an in-depth study of the experimental aspects of plate impact experiments in the particular cases of aluminum alloy 7020-T6 and AU4G1. Using impacts at different velocities $V_{0}$ ranging from $150 \mathrm{~m} / \mathrm{s}$ to $300 \mathrm{~m} / \mathrm{s}$ and with several thicknesses $l_{i}$, the spall stress was plotted against the loading time curve. Figure 6 shows the results obtained in the case of aluminum alloy 7020-T6. In this curve, the loading time $t^{c}$ and the spall stress $\sigma^{S}$ were determined by Equations [5] and [6] respectively.

\subsection{Numerical analysis}

The plate impact experiments were simulated using the explicit finite element code Europlexus (Europlexus, 2002], which is being developed by the Atomic Energy Commissary in Saclay, France for dynamic problems. A user subroutine containing the model described in Section 4 was introduced into the code using Equations [18], [23] and [26]. The numerical implementation of the model was first tested and validated on elementary cases, then used to simulate plate impact experiments. Three different meshes were used to check the mesh independence of the model (target meshed with (a) 80*24, (b) 160*48 and (c) 240*72 4-node axisymmetric elements). Aluminum alloy 7020-T6 was modelled as presented in 
Section 4. The temperature was taken as a constant equal to the room temperature because Hanim (Hanim et al., 1999) had shown that the variation of the temperature is very limited during the impact: this is due to the small strain level (only a few percents) at fracture. The duration of the simulation was $2 \mu \mathrm{sec}$.

\subsubsection{Comparison of spall stress curves}

Figure 6 shows the comparison of the predictions given by the two models (dashed lines for the model without delay effect, full line for the model with delay effect) and the experimental results (dots). A calculation is considered to represent spalling when damage has reached the critical value $D^{c}$ in a sufficiently large zone. The calculations were used in the same manner as the experiments: one point on the spall stress vs. loading time curve was obtained with one simulation corresponding to an impactor velocity such that damage reached the critical value. The simulations show that in the absence of delay effect the loading time had virtually no influence on the spall stress, even though the "viscous" effects of Klepaczko's model were included. One can observe that the viscous effects of the model affect the strain rate term, but not the damage rate term. This is clearly not the case with the delay model.

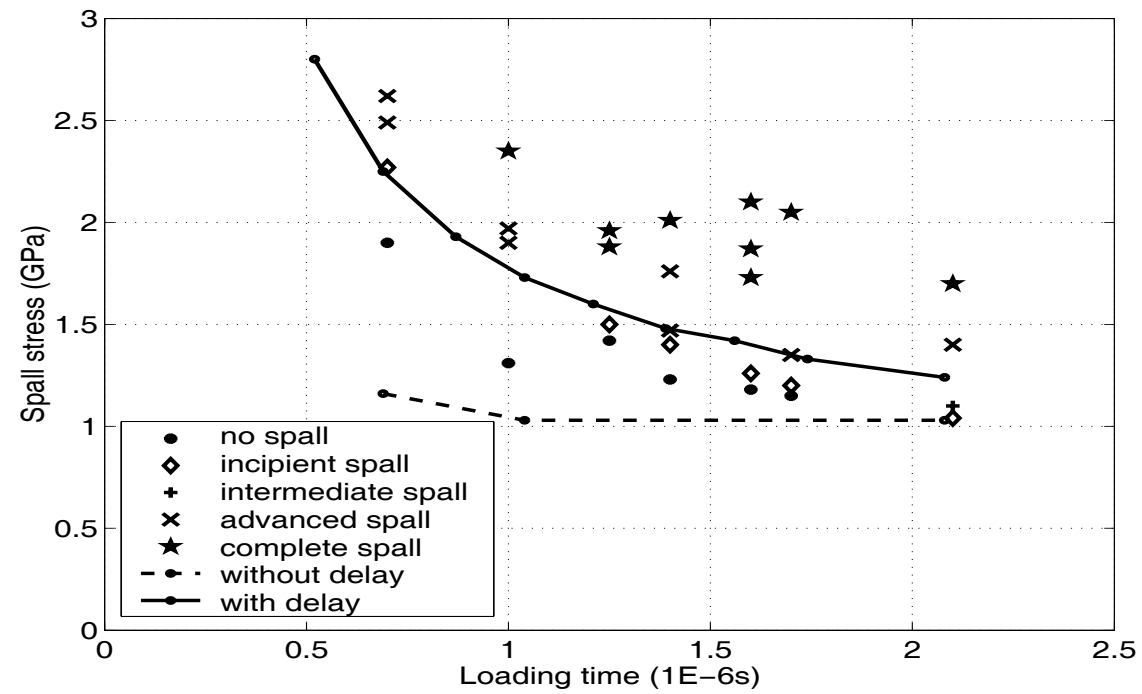

Figure 6. Spall stress as a function of the loading time. Numerical results with and without delay effect

\subsubsection{The model with delayed damage}

This section will show that the introduction of the delayed damage model enables one to interpret the experimental results. The limitation of the damage rate induces a 
dependence of the spall stress on the loading time: this observation appears clearly when one looks at the continuous curve of Figure 6 . The spall stress vs. loading time curve enables one to identify the delayed damage parameters a and $\tau_{c}$.

In the following section, we will show how the delay effect was implemented into the damage evolution law in the finite element code Europlexus in order to model the dynamic behavior of an aluminum alloy. The optimized delayed damage parameters and the corresponding calculated results will be given. Finally, a study of the mesh dependence to check the main property of the delayed damage model will be presented.

\subsubsection{Implementation of the delay effect}

The implementation of the delay effect into the model used for aluminum alloys is relatively simple. It consists simply in replacing Function $\mathrm{h}$ in Equation [23] by a new function $h^{\prime}$ calculated by the following procedure. First, one computes the damage evolution as if no delayed model were used and obtains $D_{n c}$ by forward Euler integration of the following rate equation:

$$
\dot{D}_{n c}=\frac{D^{c}}{p^{c}-p^{d}} \Omega\left(\frac{\sigma^{H}}{\sigma^{*}}\right) \dot{p}
$$

Then, one introduces the delay effect using Equation [2]. The final damage D is compared with the critical damage $D^{c}$ in order to predict failure.

\subsubsection{Identification of the parameters}

The experimental results show that the spall stress vs. loading time curve is of the hyperbolic type. Then, Parameters a and $\tau_{c}$ must be chosen so that the numerical and experimental curves match.

Furthermore, one can note that since the damage rate cannot exceed $\frac{1}{\tau_{c}}$ the minimum time $t^{r}$ to achieve complete damage is:

$$
t^{r}=\frac{D^{c}}{\dot{D}_{\max }}=D^{c} \tau_{c}
$$

Thus, if one considers that there is no damage in compression (see Equation [26]), time $D^{c} \tau_{c}$ corresponds to a vertical asymptote in Figure 6 . The higher the characteristic time $\tau_{c}$, the more the curve moves to the right. Besides, 
coefficient a enables one to highlight the difference between the uncorrected damage $D_{n c}$ and the damage with delay effect $\mathrm{D}$. The smaller the coefficient a, the greater the curvature of the spall stress vs. loading time curve. This property had already been observed by (Deü et al., 1997). With this knowledge of the influence of the two parameters of the delayed damage model, one should be able to choose them in order to fit the experimental results. A typical deformed structure is shown in Figure 7, and the damage for several mesh refinements is shown in Figure 8. After optimization, the identified parameters are found to be $\mathrm{a}=2.25$ and $\tau_{c}=0.2 \mu \mathrm{s}$. The corresponding spall stress vs. loading time curve (the continuous line named "with delay effect") is shown in Figure 6 for aluminum alloy 7020-T6.

The width of the damaged zone observed across the thickness is consistent with the prediction given by Equation [3] (1mm). The shapes of the deformed target and of the internal damaged zone are similar to those observed experimentally. Having identified the two parameters of the delayed damage model, the use of this model with these optimized parameters enables one to determine the spall stress numerically for the material being considered. One can observe that this model enables one to reproduce the hyperbolic-like increase of the spall stress when the impact velocity increases.

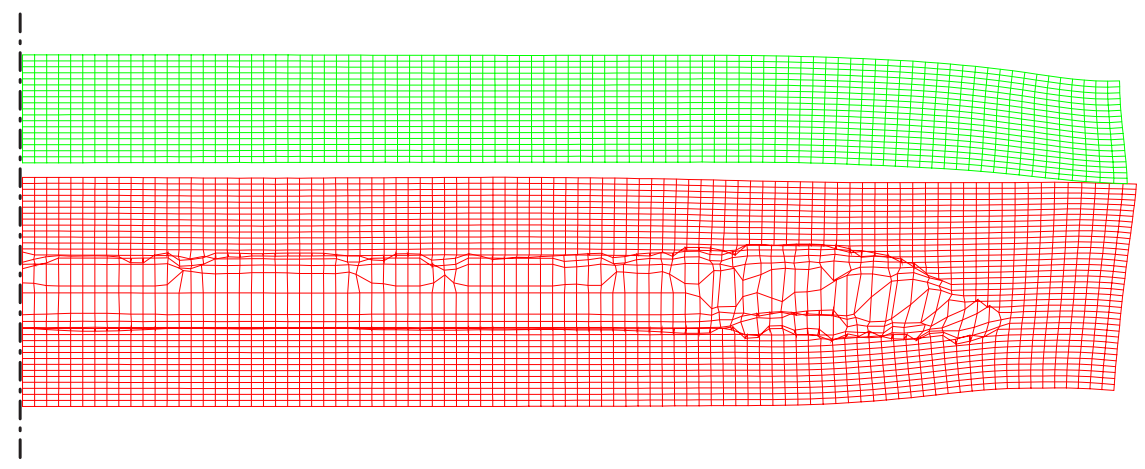

Figure 7. Deformation of the impacted plate amplified 10 times (Al 7020-T6, $\left.V_{o}=262 \mathrm{~m} / \mathrm{s}, l_{i}=3 \mathrm{~mm}\right)$, right half of the plates

An alternative means of identifying the parameters is to analyze the pull-back velocity in a spall test. In this paragraph, we apply this method to AU4G1 T4. The delayed damage model parameters are $\mathrm{a}=2$ and $\tau_{c}=0.1 \mu \mathrm{s}$ respectively. A plate impact experiment with an impact velocity of $500 \mathrm{~m} / \mathrm{s}$ was simulated with a plate/impactor thickness ratio equal to 2 . Three different plate thicknesses were tested (1, 2, 4mm). The rear-face velocity calculated without the delayed damage model is shown in Figure 9: one can observe that with this model the maximum-to-minimum stress variation is the same regardless of the plate's thickness. This is not the case when one 
takes into account the delayed damage (Figure 10). For a TA6V titanium alloy, the delayed model's predictions are consistent with experimental observations, as shown in Figure 11 in which the colored curves represent the experimental results.
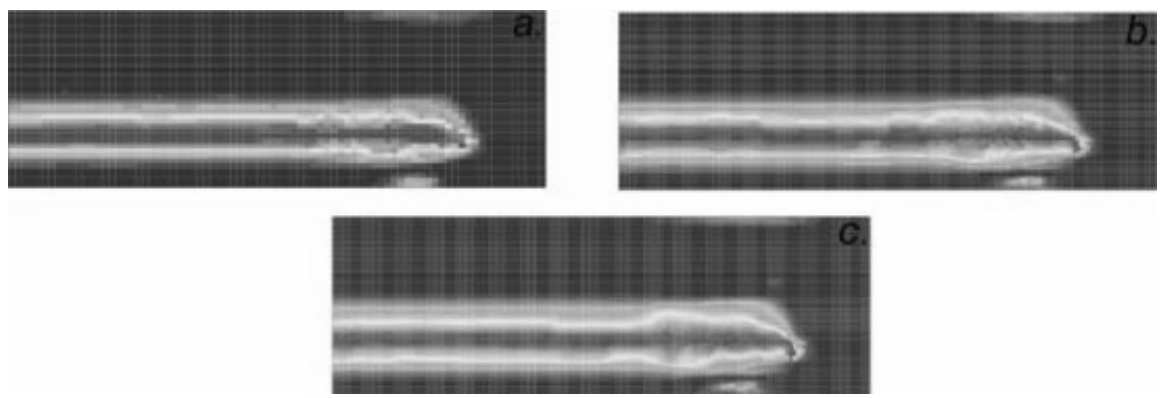

Figure 8. Damage with delay effect at the end of the simulation (7020-T6, $\left.V_{0}=262 \mathrm{~m} / \mathrm{s}, l_{i}=3 \mathrm{~mm}\right)$, right half of the target plate. The undamaged elements are in blue; the fully-damaged elements are in red. The target is meshed with a. 80*24 elements, $b .160 * 48$ elements or c. $240 * 72$ elements

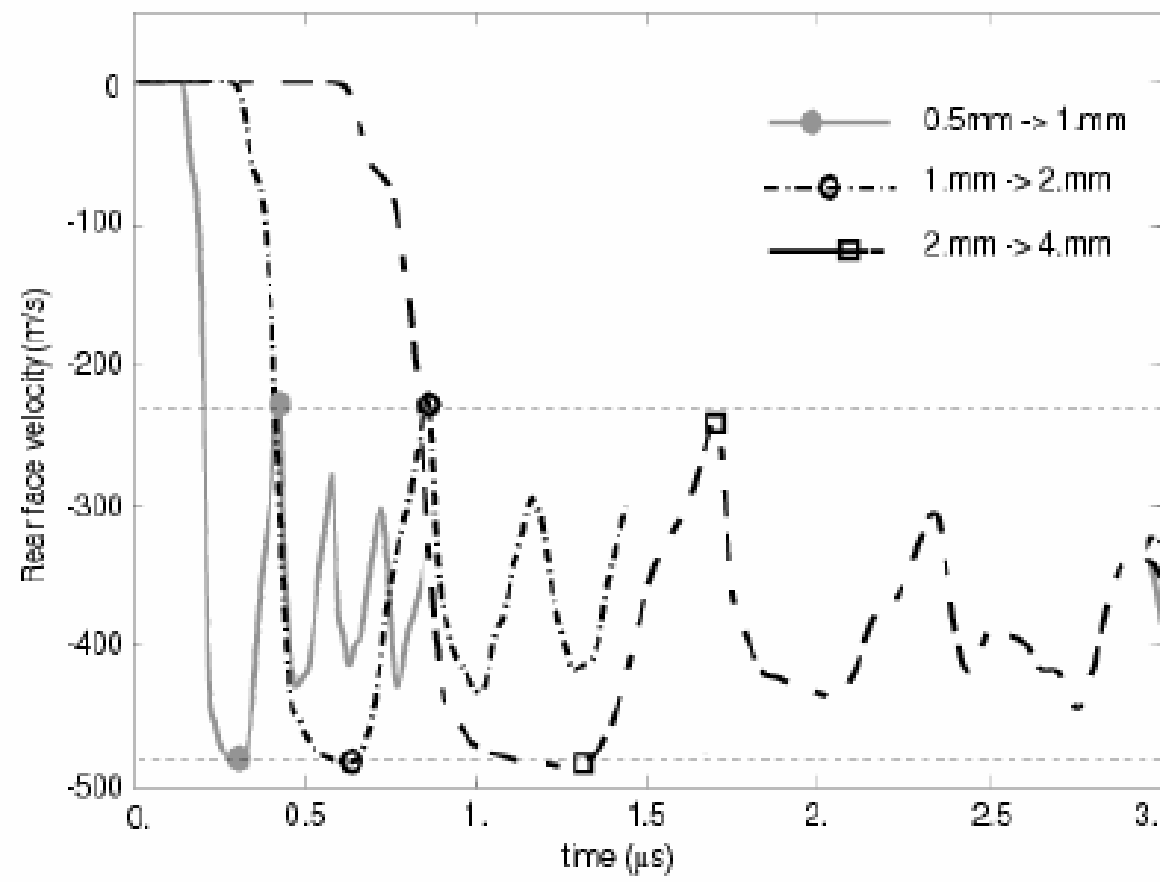

Figure 9. Calculated rear-face velocity for AU4G1 T4 without delay effect 


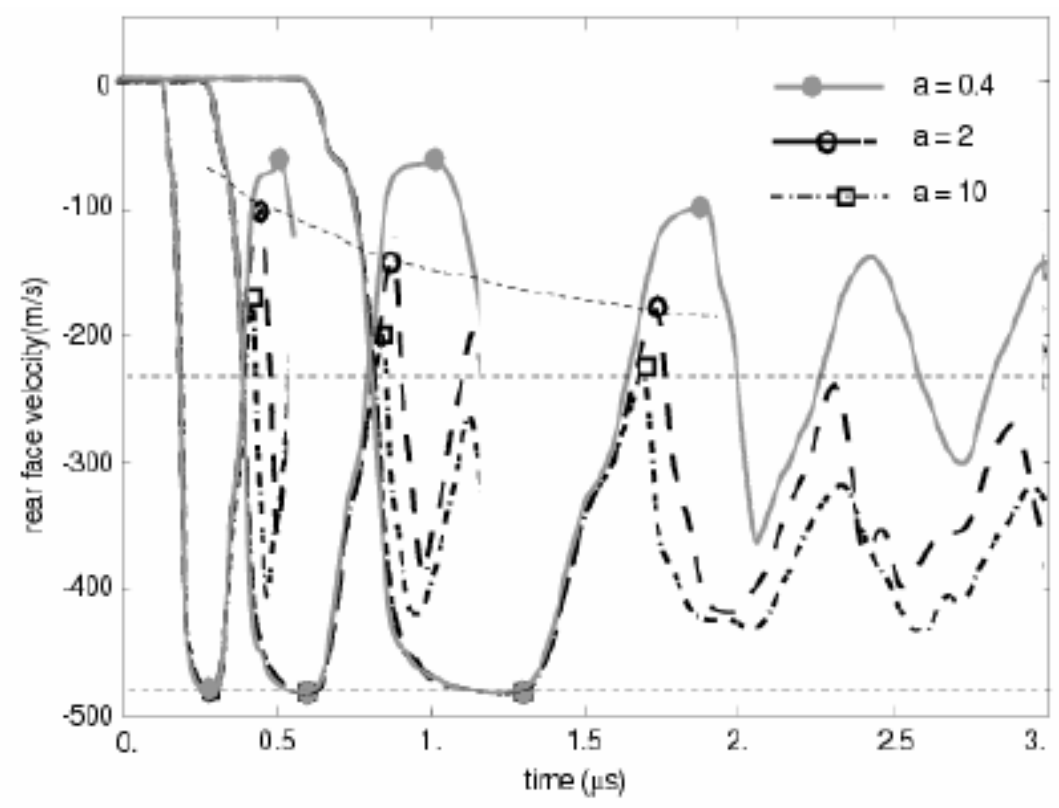

Figure 10. Calculated rear-face velocity for Au4G1 T4 with delay effect

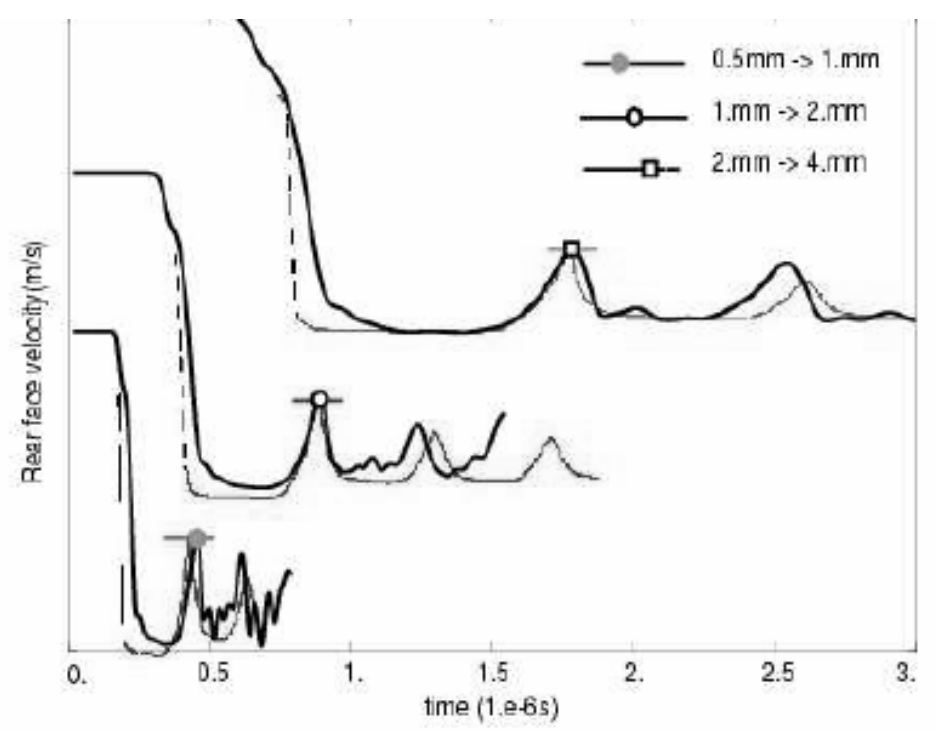

Figure 11. Comparison of rear-face velocities for TA6V, experiment vs. model with delay effect 


\subsubsection{Comparison of results for numerical localization}

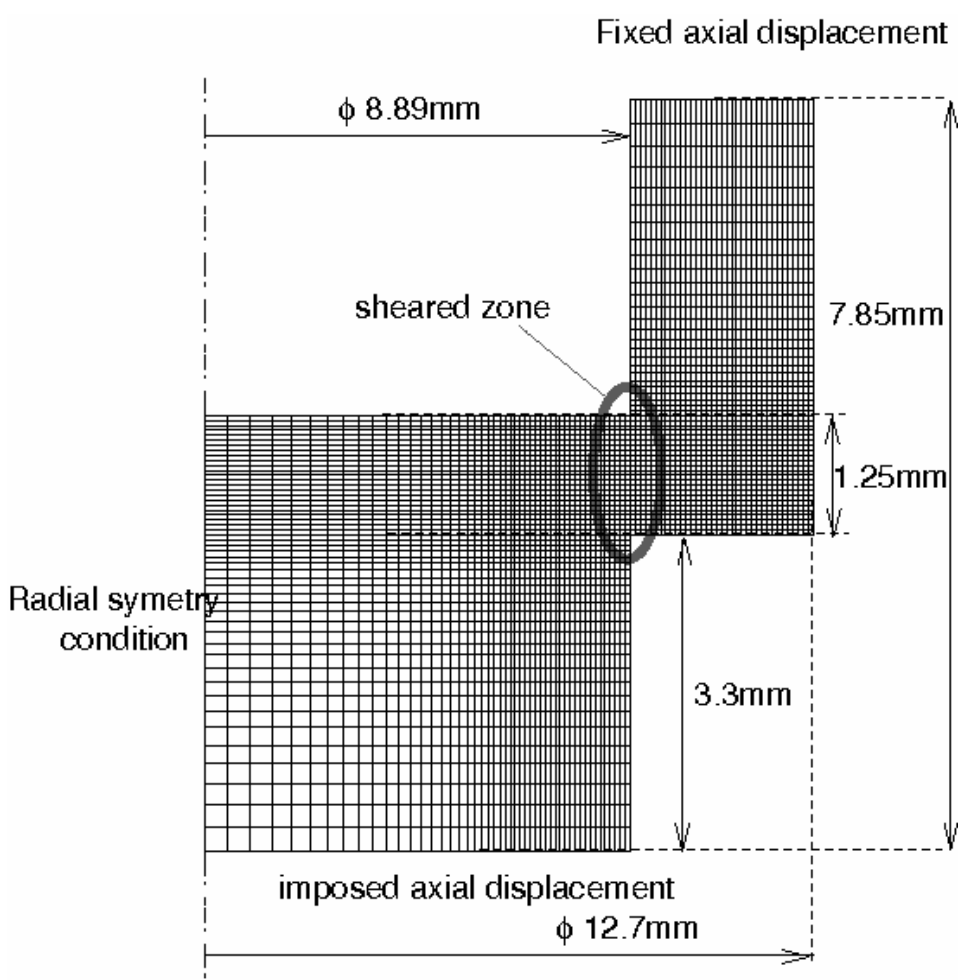

Figure 12. Geometry of the "hat" specimen

In the previous section, we observed that the delayed model enables one to represent the increase of the spall stress when the velocity of the impactor increases. In this section, we are addressing the numerical localization effect. As explained in Section 2, the delayed damage model was developed mainly to avoid mesh dependence. Let us consider the "hat" axisymmetric structure shown in Figure 12. The structure is axially fixed at the top and an axial velocity of $50 \mathrm{~m} / \mathrm{s}$ is prescribed at the base, resulting in a mean strain of about $6,000 \mathrm{~s}^{-1}$. The material is AU4G1-T4. The model identified in the previous section was used for the simulations.

A convergence study of the failure mode was performed with four meshes with 512, 4608, 51200, and 204800 4-node axisymmetric elements respectively. Figures 13 and 14, which show the damaged zone for the four meshes, indicate clearly that with the damage model without delay effect the failure mode is meshdependent (there are two cracks), whereas this is not the case with the delayed damage model, which predicts only one crack. The bifurcation of the cracks' paths could be due to the usual decision to remove the element once critical damage has been reached. 
622 Revue européenne de mécanique numérique. Volume $16-\mathrm{n}^{\circ}$ 5/2007

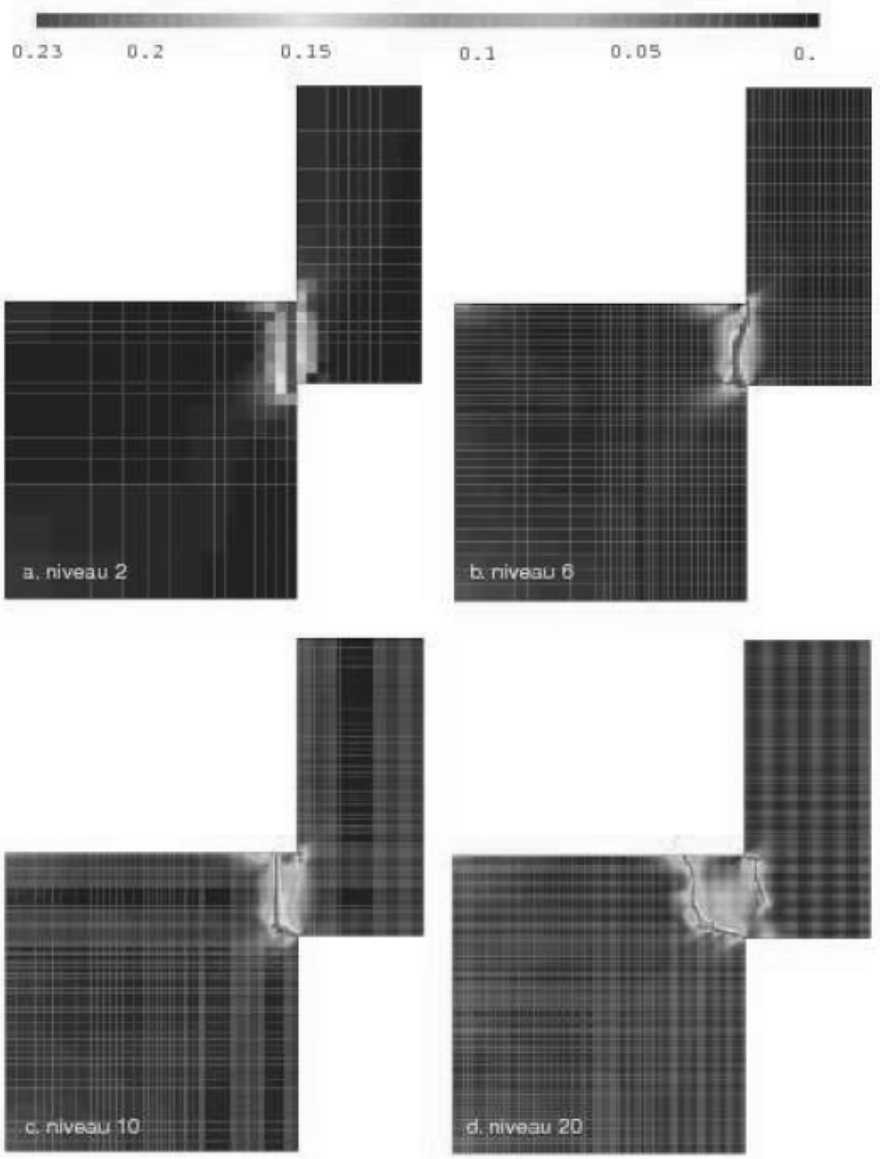

Figure13. Hat test simulation without delay effect 


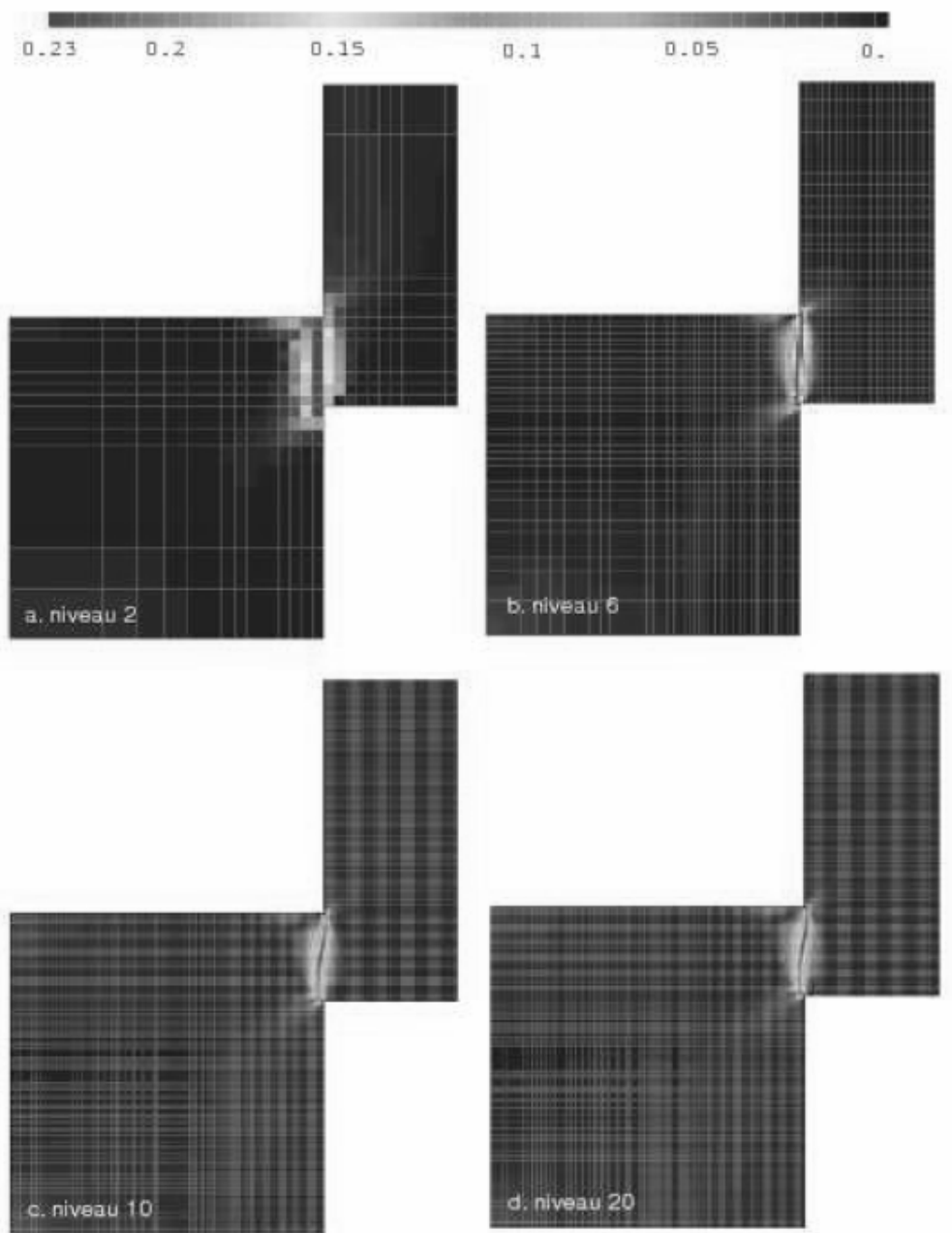

Figure 14. Hat test simulation with the delayed damage model

\section{Discussion and conclusions}

In this paper, we discussed both spalling and numerical localization. First, using a simple numerical impact experiment, we illustrated the advantage of the delayed damage model, which is to lead to mesh-independent calculated failure modes. Plate impact experiments enabled us to determine the parameters of the delayed damage model. This approach is not very difficult to implement if one has already identified 
elastic-viscoplastic damage: one only needs to add the time dependence of the model, i.e. identify the two parameters a and $\tau_{c}$.

(Chaboche et al., 1996; Lemaitre, 1996) damage models were identified and applied it to aluminum alloy 7020-T6 and to AU4G1-T4. It was found that a model which takes into account viscosity effects in the material's behavior alone and not in the failure law does not seem capable of predicting the influence of the loading time on the spall stress and is unable to avoid numerical localization if the strain rate is high. Then, we introduced the delay effect into the damage evolution law. We showed that such a model represents the classical result of the plate impact experiment properly, especially concerning the typical rear-face velocity in plate impact tests.

The delayed damage model should also help explain spall test experiments for materials other than those following J2 flow theory. The application of a bounded damage rate is not limited to metals. The model has already been used for stratified composites, but the experiments are difficult to interpret because of multiple wave reflections on the interfaces between plies (Deu et al., 1997).

\section{Acknowledgements}

The authors wish to thank Professor A.A. Lubrecht for his advice and assistance during this investigation.

\section{References}

Achon P., Comportement et tenacité d'alliages d'aluminium à haute resistance, PHD Thesis, Ecole Nationale Supérieure des Mines de Paris, Centre des Matériaux, 1994.

Allix O. et al., "A delay damage meso-model for prediction of localisation and fracture of laminates subjected to high rates loading", ECCM 99 proceedings.

Chaboche J.L. et al., Mécanique des matériaux solides, Dunod, 1996.

Chevrier P. et al., Automatisation et informatisation du fonctionnement d'un canon à gaz haute performance, et étude de l'endommagement dynamique d'un alliage d'aluminium soumis à une onde plate induite par un impact plaque sur plaque, Final eng. report, Metz University, 1994.

Chevrier P., "Discussion of fracture criteria in spall mechanics", Engng. Trans., vol 45, n 1 , 1997, p. 47-70.

Chevrier P., Mécanique et méso-mécanique de l'écaillage, essais expérimentaux et critères de rupture: étude d'un alliage d'aluminium et d'un acier de blindage, PHD Thesis, Université de Metz, Laboratoire de Physique et Mécanique des Matériaux de Metz, 1998. 
Chevrier P. et al., "Spall fracture: mechanical and microstructural aspects", Eng. Fract. Mech., vol. 63, 1999, p. 273-294.

Curran D.R., Dynamic failure of solids, Physics reports, vol. 147, 1987, p. 253-388.

Deu J.F. et al., "Delayed-damage modelling for fracture prediction of laminated composites under dynamic loading", Engng. Trans., vol. 45, n 1, 1997, p. 29-46.

EuroPlexus, A computer program for the finite element simulation of fluid-structure systems under dynamic loading, User's manual, CEA Saclay, CEA/DEN/SEMT/DYN, 2002.

Gurson A.L., "Continuum theory of ductile rupture by void nucleation and growth: Part I Yied criteria and flow rules for porous ductile media", J. of the Mech. and Phys. of Sol., vol. 17, 1977, p. 201-217.

Hanim S. et al., "Numerical study of spalling in an aluminium alloy 7020-T6", Int. J. of Imp. Eng., vol. 22, 1999, p. 649-673.

Ikkurthi V. R., "Use of different damage models for simulating impact-driven spallation in metal plates", Int. J. of Impact Engineering, vol. 47, n 11, 2004, p. 4814-4824.

Klepaczko J.R., "A practical stress-strain-strain rate-temperature constitutive relation of the power form”, J. Mech. Working Technology, vol. 15, 1987, p. 143-166.

Ladevèze P., "A damage computational method for composite structures", Computers and Structures, vol. 44, 1992, p. 79-87.

Ladevèze P. et al., "A mesomodel for localisation and damage computation in laminates", Comput. Meth. Appl. Mech. Engrg., vol. 183, 2000, p. 105-122.

Lemaitre J., A course on damage mechanics, Springer, Paris, 1996.

Seaman L., "Dynamic failure of solids", Phys Rep, vol. 147, n 5-6, 1987, p. 253-388.

Suffis A. et al., «Modèle d'endommagement à effet retard, étude numérique et analytique de l'évolution de la longueur caractéristique", Revue Européenne des Eléments Finis, vol 11, n $5,2002$.

Suffis A. et al., "Damage Model with Delay Effect: Analytical and Numerical Studies of the evolution of the characteristics length", Int. J. of Sol. and Stru., vol. 40, 2003, p. 34633476 .

Suffis A., Développement d'un modèle d'endommagement à taux de croissance contrôlée pour la simulation robuste de ruptures sous impact, PHD Thesis, INSA-Lyon 2004.

Tuler F.R., "A criterion for the time dependence of dynamic fracture", Int. J. of Fract. Mech., vol. 4, 1968, p. 431-437.

Zerilli F.J. et al., "Dislocation mechanics based analysis of material dynamics behaviour: enlaced ductility, deformation twinning, shock deformation, shear instability, dynamic recovery", J. of Phys, IV France 7, vol. 61, 1977, C3-637. 
